\title{
Clinical features of Parkinson's disease with and without rapid eye movement sleep behavior disorder
}

\author{
Ye Liu ${ }^{1 \dagger}$, Xiao-Ying Zhu' ${ }^{1 \dagger}$, Xiao-Jin Zhang ${ }^{1}$, Sheng-Han Kuo ${ }^{2}$, William G. Ondo ${ }^{3}$ and Yun-Cheng Wu ${ }^{1 *}$
}

\begin{abstract}
Background: Rapid eye movement sleep behavior disorder (RBD) and Parkinson's disease (PD) are two distinct clinical diseases but they share some common pathological and anatomical characteristics. This study aims to confirm the clinical features of RBD in Chinese PD patients.

Methods: One hundred fifty PD patients were enrolled from the Parkinson's disease and Movement Disorders Center in Department of Neurology, Shanghai General Hospital from January 2013 to August 2014. This study examined PD patients with or without RBD as determined by the REM Sleep Behavior Disorder Screening Questionnaire (RBDSQ), assessed motor subtype by Unified PD Rating Scale (UPDRS) III at "on" state, and compared the sub-scale scores representing tremor, rigidity, appendicular and axial. Investigators also assessed the Hamilton Anxiety Scale (HAMA), Hamilton Depression Scale (HAMD), Mini-Mental State Examination (MMSE), Clinical Dementia Rating (CDR), and Parkinson's disease Sleep Scale (PDSS).
\end{abstract}

Results: One hundred fourty one PD patients entered the final study. 30 (21.28\%) PD patients had probable RBD (pRBD) diagnosed with a RBDSQ score of 6 or above. There were no significant differences for age, including age of PD onset and PD duration, gender, smoking status, alcohol or coffee use, presence of anosmia or freezing, UPDRS III, and $\mathrm{H}-\mathrm{Y}$ stages between the $\mathrm{pRBD}^{+}$and $\mathrm{pRBD}^{-}$groups. $\mathrm{pRBD}{ }^{+}$group had lower MMSE scores, higher PDSS scores, and $\mathrm{pRBD}^{+}$PD patients had more prominent proportion in anxiety, depression, constipation, hallucination and a greater prevalence of orthostatic hypotension.

Conclusion: $\mathrm{PRBD}^{+}$PD patients exhibited greater changes in non-motor symptoms. However, there was no increase in motor deficits.

Keywords: Rapid eye movement sleep behavior disorder, Parkinson's disease, Depression, Cognitive decline, Orthostatic hypotension, Motor deficits

\section{Background}

Rapid eye movement sleep (REM) is characterized by decreased or absent muscle tone (atonia), desynchronization of the electroencephalogram, with the presence of saw tooth waves, and autonomic instability. Rapid eye movement sleep behavior disorder (RBD) is a form of parasomnia during which patients develop limb or body movements, which

\footnotetext{
* Correspondence: yunchw@medmail.com.cn

Ye Liu and Xiao-Ying Zhu are co-first authors

${ }^{\dagger}$ Equal contributors

'Department of Neurology, Shanghai General Hospital, Shanghai Jiao Tong University School of Medicine, No.100, Haining Road, Shanghai 200080,

People's Republic of China

Full list of author information is available at the end of the article
}

correlate with dream enactment behavior. The abnormal physiology of RBD is loss of muscle atonia (paralysis) during otherwise intact REM sleep [1].

The standard RBD diagnostic criteria are based on the 2nd edition of the International Classification of Sleep Disorders (ICSD) [2], and polysomnology (PSG) is necessary for a definitive diagnosis. Nomura et al., determined that RBD rapid screening questionnaire (RBDSQ), which is completed by the patient, had a sensitivity of $84.2 \%$ and specificity of $96.2 \%$ to diagnose RBD when compared with standard RBD diagnostic criteria using PSG in PD at a cut off of 6 points (total score of RBDSQ is 13, and a score of 5 is the cut-off point for healthy individuals) [3]. Chahine 
et al. investigated the use of the RBDSQ plus Mayo Sleep questionnaire 1 (MSQ1) compared with PSG in PD patients. They found sensitivity was highest when the questionnaires were used in combination while specificity was highest for the RBDSQ used alone at a cut-off point of 7 [4]. Shen SS used the RBDSQ to diagnose RBD in Chinese patients, compared with PSG, and found a cutoff points at 6 had the best specificity and sensitivity [5].

RBD has a close relationship with neurodegenerative diseases, especially those with $\alpha$-synucleinopathy pathology such as PD, dementia with Lewy bodies (DLB) and multiple system atrophy (MSA) [1, 6]. Recently, studies have indicated that PD patients with RBD might have some specific clinical features more commonly than those without RBD. Although data is mixed, PD patients with RBD have been reported to have worse decision-making [7], cognitive impairment [6], freezing, falls and rigidity $[8,9]$. They also have a higher prevalence of orthostatic hypotension $(\mathrm{OH})[8]$ and visual hallucinations $(\mathrm{VH})$ [10]. However, there are no detailed data in PD patients with RBD in China. In this paper, we investigated the clinical features of PD patients with RBD in a tertiary referral center in China. The present study focused on the characteristics of motor and non-motor symptoms of PD with RBD compared to PD without RBD.

\section{Methods}

\section{Patient selection}

One hundred fifty PD patients were enrolled from the Parkinson's disease and Movement Disorder Center in the Department of Neurology, Shanghai General Hospital, Shanghai Jiao Tong University, Shanghai, China from January 2013 to August 2014. The diagnosis of PD was made by two movement disorder specialists according to the UK Parkinson's Disease Society (UKPDS) Brain Bank Criteria. Patients with severe dementia $(C D R \geq 2)$, or other central nervous system disorders were excluded from this study. The study was approved by the Institution's Ethics Committee and all recruited patients consented to participate in the study.

\section{Patient evaluation}

Of 150 patients, 5 patients were excluded because of probable DLB, 3 patients had probable MSA, and 1 patient had vascular Parkinsonism. A total of 141 patients were enrolled in the study. Patient evaluation was performed by movement disorder specialists. Parkinsonism staging was evaluated according to the Hoehn \& Yahr staging scale. Part III of the Unified PD Rating Scale (UPDRS III) was performed during the "on" state. Motor subtype was analyzed by predominance of tremor or rigidity (UPDRS subscores) and predominance of limb or axial features (UPDRS sub-scores) [9], Hamilton Anxiety Scale (HAMA; cut-off point $\geq 8$ ), Hamilton depression Scale (HAMD; cutoff point $\geq 8$ ) and Mini-Mental State Examination (MMSE) were used to evaluate the patient's mood and cognitive state. The patients whose MMSE score was lower than 17 (illiteracy) or lower than 20 (primary school level) or 24 (higher than middle school cultural level) [11] were considered to have dementia and were evaluated using the Clinical Dementia Rating (CDR). Patients whose CDR was higher than 2 were excluded from the study. Investigators used the REM Sleep Behavior Disorder Screening Questionnaire(RBDSQ)to detect clinical probable RBD (pRBD). We set the RBDSQ cut-off point at a score of 6 according to the highest sensitivity and specificity determined by a previous study [5]. The Parkinson's disease Sleep Scale (PDSS) was used to evaluate patient's sleep quality. Orthostatic hypotension $(\mathrm{OH})$ was screened using a simple question:"Do you feel dizziness or weakness when you stand up?" If the answer was "yes", a blood pressure test from the supine to standing position was checked, a fall in systolic blood pressure of $\geq 20 \mathrm{mmHg}$, or in diastolic blood pressure of $\geq 10 \mathrm{mmHg}$, was diagnosed as $\mathrm{OH}$. Other items including smoking, alcohol and coffee consumption, hyposmia or anosmia, constipation were also documented by question. Patients continued with their prescribed treatment regimen, they used anti-Parkinson drugs, antihypnotics or anti-depressants as necessary.

\section{Statistical analysis}

The data were analyzed using the Statistical Package for Social Sciences (SPSS) 19(IBM Co., USA). The data is presented as mean, counts and percentages, and the adjusted difference in means. Analysis of descriptive variables was performed using two-tailed t tests. MannWhitney $U$ tests and $X^{2}$ tests were used where appropriate. A P-P plot was used to test normal distribution. A $p$ value $<0.05$ was considered to be significant.

\section{Results \\ Demographics}

Among 141 patients, 74 were male (52.48\%): 18 male $(60 \%)$ in $\mathrm{pRBD}^{+}$group, and 56 male $(50.45 \%)$ in $\mathrm{pRBD}^{-}$group $(p=0.655)$. Thirty patients $(21.28 \%)$ were diagnosed with probable RBD (pRBD) based on a RBD screening questionnaire score $\geq 6$. If the cut-off score was set at 7 or 5 , the incidence of RBD was $17.02 \%$ and $26.24 \%$ respectively, with little difference in the clinical features (Table 3). The mean age in the pRBD $^{+}$group was $68.33 \pm 8.76$ versus $69.32 \pm 9.75$ years in the $\mathrm{pRBD}^{-}$group $(p=0.618)$. Mean PD duration years is $4.13 \pm 4.216$ in $\mathrm{pRBD}^{+}$and $4.65 \pm 3.570$ in $\mathrm{pRBD}^{-}$group $(p=0.5)$. Smoking, alcohol and coffee consumption were infrequent in both groups (NS). There was no difference between the numbers of patients who took levodopa or dopamine agonists, and the levodopa equivalent dosage was similar in both 
Table 1 Epidemiological characteristic of PD patients

\begin{tabular}{|c|c|c|c|}
\hline & pRBD+ & pRBD- & $p$ \\
\hline Patients number $(n)$ & 30 & 111 & \\
\hline Age $(y r)$ & $68.33 \pm 8.76$ & $69.32 \pm 9.75$ & 0.618 \\
\hline PD duration (yr) & $4.13 \pm 4.22$ & $4.65 \pm 3.57$ & 0.500 \\
\hline Gender, male, $n$ (\%) & $18(60.00)$ & $56(50.45)$ & 0.655 \\
\hline Smoking, $n(\%)$ & $4(13.3)$ & $11(9.91)$ & 0.525 \\
\hline Alcohol, $n(\%)$ & 0 & $9(8.11)$ & 0.204 \\
\hline Coffee, $n(\%)$ & $1(3.33)$ & $5(4.50)$ & 1.0 \\
\hline $\begin{array}{l}\text { Levodopa dose } \\
\text { equivalent (mg/day) }\end{array}$ & $353.53 \pm 236.10$ & $339.10 \pm 272.08$ & 0.531 \\
\hline Levodopa dose (mg/day) & $325.00 \pm 205.84$ & $285.15 \pm 250.09$ & 0.221 \\
\hline Levodopa years & $2.59 \pm 3.08$ & $3.03 \pm 3.31$ & 0.577 \\
\hline Dopa agonist dose (mg/day) & $38.23 \pm 51.01$ & $33.19 \pm 45.12$ & 0.931 \\
\hline Dopa agonist years & $0.91 \pm 1.52$ & $4.41 \pm 29.21$ & 0.479 \\
\hline TCA(Deanxit), $n(\%)$ & $1(0.033)$ & $1(0.009)$ & $>0.90$ \\
\hline Trihexyphenidyl, $n$ (\%) & $4(0.133)$ & $18(0.162)$ & $>0.90$ \\
\hline SSRI, $n(\%)$ & $2(0.067)$ & $3(0.027)$ & $>0.75$ \\
\hline BNZ, n (\%) & $6(0.2)$ & $3(0.027)$ & $<0.005$ \\
\hline
\end{tabular}

SSRI Selective Serotonin Reuptake Inhibitor

$B N Z$ Benzodiazepines, n: number

The age, mean PD duration years, smoking, alcohol and coffee consumption rates, levodopa had no difference between two groups. However, the pRBD ${ }^{+}$group had greater antidepressant and antihypnotic use

groups. However, the $\mathrm{pRBD}^{+}$group had greater antidepressant and anti-hypnotic use (Table 1).

\section{Non-motor symptoms}

$\mathrm{pRBD}^{+}$PD patients had significantly higher rates of anxiety $(60 \%$ vs $22.52 \%,<0.001)$ and depression $(63.33 \%$ vs $24.32 \%, p<0.001$ ) (Table 2), and a higher mean PDSS score $(17.50 \pm 9.60$ vs $11.70 \pm 8.55, \mathrm{p}<0.001)$ (Table 2). The $\mathrm{pRBD}^{+}$group had lower mean MMSE score $(25.30 \pm 3.91$ vs. $26.75 \pm 3.97, p=0.017$ ) (Table 3 ). When educational status was taken into account, a diagnosis of dementia was not significantly difference between two groups $(16.67 \%$ vs $11.71 \%, p=0.538$ ) (Table 2). Constipation and $\mathrm{OH}$ were more prominent in the $\mathrm{pRBD}^{+}$group (Table 2 ).

There was no difference in the incidence of anosmia in $\mathrm{pRBD}^{+}$vs $\mathrm{pRBD}^{-}$group $(46.67 \%$ vs $35.16 \%, p=0.247$ ) (Table 2). From PDSS item 7, we found that there were 13 patients who had visual hallucinations, $20 \%$ (6) in $\mathrm{pRBD}^{+}$ group and $6.3 \%$ (7) in $\mathrm{pRBD}^{-}$group $(p=0.032)$ (Table 2).

\section{Motor symptoms}

The UPDRS III score was similar in the $\mathrm{pRBD}^{+}$and $\mathrm{pRBD}^{-}$ groups $(26.93 \pm 14.62$ vs $23.68 \pm 15.93, p=0.174)$. Mean $\mathrm{H}^{-}$ $\mathrm{Y}$ stage was also similar between the two groups $(2.40 \pm$ 0.90 vs $2.26 \pm 0.90, p=0.299$ ). When limb scores in UPDRS III was compared with axial scores, the ratio showed no difference between $\mathrm{pRBD}^{+}$and $\mathrm{pRBD}^{-}$groups $(5.67 \pm 4.22$ vs $6.17 \pm 4.61, p=0.734)$. When rigidity scores in UPDRS III
Table 2 Clinical characteristics of PD patients

\begin{tabular}{llll}
\hline & pRBD + & pRBD- & $p$ \\
\hline Hoehn \& Yahr stage & $2.40 \pm 0.90$ & $2.26 \pm 0.90$ & 0.299 \\
UPDRS III(total) & $26.93 \pm 14.62$ & $23.68 \pm 15.93$ & 0.174 \\
Bradykinesia (23-26) & $8.87 \pm 7.05$ & $7.40 \pm 6.11$ & 0.292 \\
Freezing, $n$ (\%) & $8(26.7)$ & $25(22.52)$ & 0.750 \\
LcompareA & $5.67 \pm 4.22$ & $6.17 \pm 4.61$ & 0.734 \\
RcompareT & $3.01 \pm 4.06$ & $1.79 \pm 2.08$ & 0.320 \\
PDSS & $17.50 \pm 9.60$ & $11.70 \pm 8.55$ & $<0.001$ \\
MMSE & $25.30 \pm 3.91$ & $26.75 \pm 3.97$ & 0.017 \\
HAMA & $7.53 \pm 5.51$ & $4.53 \pm 6.38$ & 0.002 \\
HAMD & $10.83 \pm 8.60$ & $5.39 \pm 7.12$ & $<0.001$ \\
Hallucination, $n$ (\%) & $6(20.00)$ & $7(6.30)$ & 0.032 \\
Dementia, $n$ (\%) & $5(16.67)$ & $13(11.71)$ & 0.538 \\
Anxiety, $n$ (\%) & $18(60.00)$ & $25(22.52)$ & $<0.001$ \\
Depression, $n$ (\%) & $19(63.33)$ & $27(24.32)$ & $<0.001$ \\
Hyposmia, \% & 46.67 & 35.16 & 0.247 \\
Constipation, \% & 80.00 & 57.66 & 0.033 \\
Orthostatic hypotension, $n$ (\%) & $7(23.33)$ & $7(6.30)$ & 0.012 \\
\hline Lcompar & &
\end{tabular}

LcompareA (UPDRS III): UPDRS score in limb compare with axial; RcompareT (UPDRS III): UPDRS score in rigidity compare with tremor; $n$ Number The $\mathrm{pRBD}^{+} \mathrm{PD}$ patients had significantly higher rates of anxiety, depression and visual hallucinations rates. And they had a higher mean PDSS score. Dementia was not significantly difference between two groups. Constipation and $\mathrm{OH}$ were more prominent in the $\mathrm{pRBD}^{+}$group 
Table 3 The clinical difference of $\mathrm{RBD}^{+}$vs $\mathrm{RBD}^{-}$patients while RBDSQ cutoff at 7, 6 and 5 in present study

\begin{tabular}{llll}
\hline & 7 score & 6 score & 5 score \\
\hline RBD incidence, $n$ (\%) & $24(17.02)$ & $30(21.28)$ & $37(26.24)$ \\
Age & - & - & - \\
PD duration & - & - & - \\
Gender & - & - & - \\
Dopamine use & - & - & - \\
Hypnosmia & - & - & - \\
Freezing & - & - & - \\
Hallucination & + & + & - \\
Constipation & + & + & + \\
HAMA & + & + & + \\
HAMD & + & + & + \\
PDSS & + & + & + \\
MMSE & + & + & + \\
Orthostatic hypotension & + & - & - \\
UPDRS III (total) & - & - & - \\
Bradykinesia (23-26) & - & - & - \\
LcompareA & - & - & + \\
RcompareT & - & - & - \\
\hline
\end{tabular}

If the cut off score was set at 7,6, or 5, the clinical features had little difference. If the cut off score was set at 5 score, the hallucination rates would have no difference in two groups instead. The other features were same in the three condition were compared with resting tremor score, the ratio was also not significantly different $(3.01 \pm 4.06$ vs $1.79 \pm 2.08, p=$ 0.32). Overall there was no difference between $\mathrm{pRBD}^{+}$ group and $\mathrm{pRBD}^{-}$group for motor severity and motor subtype (Table 2). Freezing was also not different between the two groups $(26.7 \%$ vs $22.52 \%, p=0.75)$.

\section{Discussion}

Probable RBD is common in early PD and predicts future cognitive decline, particularly in attention and memory domains [12]. The pedunculopontine nucleus (PPN) and locus cerulean (LC)/ dorsal subcoeruleus (subCD) are compromised in both PD and RBD [13-15]. Autopsy studies show that the loss of cholinergic neurons of the PPN in PD has a significant negative correlation with the modified Hoehn and Yahr stage [16], and contribute to freezing and falls $[16,17]$. Dysfunction of the PPN relates to visual hallucination (VH) [15]. A resting-state functional connectivity MRI (rs-fcMRI) study in RBD patient showed reduced connection between lateral geniculate nuclei LGN and visual association cortex [18]. PD patients with probable RBD showed smaller volumes than patients without RBD and than healthy controls in the pontomesencephalic tegmentum where cholinergic, GABAergic and glutamatergic neurons are located. It is additionally associated with

Table 4 Clinical features of PD with RBD patients

\begin{tabular}{|c|c|c|c|c|c|c|c|}
\hline & Present study & Sixel-Doring 2014 & Yoritaka 2009 & Vibha 2011 & Romenets 2012 & Sommerauer 2014 & Rolinski 2014 \\
\hline Patients $(n)$ & 141 & 158 & 150 & 134 & 98 & 59 & 475 \\
\hline $\mathrm{RCP}$ & 6 & no & b & b & no & no & 5 \\
\hline RBD (\%) & 21.28 & 51.27 & - & 19.4 & - & - & 47.2 \\
\hline Male pro & - & - & + & - & + & - & + \\
\hline older & - & - & + & - & + & - & - \\
\hline MMSE & + & - & - & - & no & no & + \\
\hline PDSS & + & no & no & + & no & no & a \\
\hline Depression/anxiety & + & no & no & - & + & no & + \\
\hline Dementia & - & no & - & no & ex & no & + \\
\hline Hallucination & + & no & - & + & - & no & + \\
\hline $\mathrm{N}$-Tremor prominent & - & - & - & - & + & - & - \\
\hline Axial/limb ratio & - & - & no & no & - & no & + \\
\hline Constipation & + & no & + & no & - & no & + \\
\hline Hyposmia & - & - & no & no & - & no & - \\
\hline $\mathrm{OH}$ & + & no & - & no & + & no & + \\
\hline PSG & $\mathrm{N}$ & Y & $\mathrm{N}$ & $\mathrm{N}$ & Y & Y & $\mathrm{N}$ \\
\hline
\end{tabular}

-: no difference, +: significant difference

Ex: dementia patient was excluded

Male pro Male prominent

No: no data

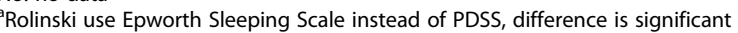

boritaka and Vibha use minimum clinical criteria of ICSD for RBD diagnosis instead

$N$ No

$\mathrm{N}$-tremor prominent Non-tremor prominent

$\mathrm{OH}$ Orthostatic hypotension

$Y$ Yes

$R C P$ RBDSQ cutoff points

Overall motor symptoms and signs were similar but we found significant difference between the two groups in many aspects of non-motor symptoms, including MMSE performance, visual hallucinations, depression, anxiety, orthostatic hypotension and constipation. Except for constipation, these results are consistent with most previous studies 
more widespread atrophy in other subcortical and cortical regions [19]. The basal ganglia activity is changed across the sleep-wake cycle in RBD [20]. The appearance of RBD in PD may be related to regional gray matter changes in the left posterior cingulate and hippocampus but not localized to the brain stem [21].

Our study had compared motor and non-motor symptoms in PD patients with and without RBD. The results were similar if we used a RBDSQ cut off point at 6 or 7 (Table 3). Overall motor symptoms and signs were similar but we found significant difference between the two groups in many aspects of non-motor symptoms, including MMSE performance, visual hallucinations, depression, anxiety, orthostatic hypotension and constipation. Except for constipation, these results are consistent with most previous studies (detailed in Table 4) [7, 22-24]. Our findings are consistent with some studies that there are no difference between the motor symptoms [13, 23], however, some previous studies indicated that pRBD+ patients showed much more worse in the gait, balance or increased dyskinesia (Table 4) [8, 9, 22, 25].

In summary, this study systematically investigated the clinical features of PD patients with RBD. There are several potential weaknesses. We used the RBDSQ to detect RBD in PD patients which is easier and more readily available than PSG. The sample size is relatively small and it might have given false negatives and false positives for diagnosing RBD without PSG. We diagnosed the patient with anosmia and constipation only based on self-report and not using objective examination. Neurological image and electrophysiology will be valuable for further study.

\section{Conclusions}

The present study demonstrated that there were no significant differences in motor deficits in $\mathrm{pRBD}^{+} \mathrm{PD}$ patients, while the non-motor symptoms are prominent, such as mood, sleep, constipation, cognition and orthostatic hypotension. However, further studies and laboratory tests are needed to improve the understanding of RBD in PD.

\begin{abstract}
Abbreviations
CDR: Clinical Dementia Rating; DLB: Dementia with Lewy bodies; HAMA: Hamilton Anxiety Scale; HAMD: Hamilton Depression Scale; ICSD: International Classification of Sleep Disorders; LC: Locus cerulean; MMSE: Mini-Mental State Examination; MSA: Multiple system atrophy; MSQ1: Mayo Sleep questionnaire 1; $\mathrm{OH}$ : Orthostatic hypotension; PD: Parkinson's disease; PDSS: Parkinson's disease Sleep Scale; PPN: Pedunculopontine nucleus; pRBD: Probable RBD; PSG: Polysomnology; RBD: Rapid eye movement sleep behavior disorder; RBDSQ: REM Sleep Behavior Disorder Screening Questionnaire; subCD: Dorsal subcoeruleus; $\mathrm{VH}$ : Visual hallucinations
\end{abstract}

\section{Acknowledgments}

This work was supported by the National Natural Science Foundation of China (NSFC) $(81171205,81371410)$ and the Biomedical Multidisciplinary Program of Shanghai Jiao Tong University (YG2014MS31, YG2015QN21, YG2016QN25).

\section{Funding}

National Natural Science Foundation of China, Biomedical Multidisciplinary Program of Shanghai Jiao Tong University.

\section{Availability of data and materials}

All the data mentioned in this article are available on published article.

\section{Authors' contributions}

$Y L, X-Y Z$ and $Y-C W$ mainly designed and drafted the manuscript. WGO and $Y$-CW were involved in critically revising the manuscript and provided intellectual thoughts. X-JZ attended the survey. S-HK modified the manuscript. All authors read and approved the final manuscript to be published.

Ethics approval and consent to participate

Ethics approval was permitted by ethics committee of Shanghai Jiao Tong University affiliated Shanghai General Hospital. Approval paper number is

2015KY128. The consents to participate are stored in Ethics office.

\section{Consent for publication}

Not applicable.

\section{Competing interests}

The authors declare that they have no competing interests.

\section{Author details}

${ }^{1}$ Department of Neurology, Shanghai General Hospital, Shanghai Jiao Tong University School of Medicine, No.100, Haining Road, Shanghai 200080, People's Republic of China. ${ }^{2}$ Department of Neurology, College of Physicians and Surgeons, Columbia University, New York, USA. ${ }^{3}$ Methodist Neurological Institute, Houston, TX, USA.

Received: 17 August 2017 Accepted: 4 December 2017

Published online: 21 December 2017

\section{References}

1. Frenette E. REM sleep behavior disorder. Med Clin North Am. 2010;94:593-614.

2. Sateia MJ. International classification of sleep disorders-third edition: highlights and modifications. Chest. 2014;146:1387-94.

3. Nomura T, Inoue Y, Kagimura T, Uemura Y, Nakashima K. Utility of the REM sleep behavior disorder screening questionnaire (RBDSQ) in Parkinson's disease patients. Sleep Med. 2011;12:711-3.

4. Chahine LM, Daley J, Horn S, Colcher A, Hurtig H, Cantor C, Dahodwala N. Questionnaire-based diagnosis of REM sleep behavior disorder in Parkinson's disease. Mov Disord. 2013;28:1146-9.

5. Shen SS, Liu CF. [validation study of related questionnaires of REM sleep behavior disorder]. Graduation dissertation. China: Soochow Universtiy; 2012.

6. Vendette M, Gagnon JF, Decary A, Massicotte-Marquez J, Postuma RB, Doyon J, Panisset M, Montplaisir J. REM sleep behavior disorder predicts cognitive impairment in Parkinson disease without dementia. Neurology. 2007:69:1843-9.

7. Delazer M, Hogl B, Zamarian L, Wenter J, Ehrmann L, Gschliesser V, Brandauer E, Poewe W, Frauscher B. Decision making and executive functions in REM sleep behavior disorder. Sleep. 2012;35:667-73.

8. Romenets SR, Gagnon JF, Latreille V, Panniset M, Chouinard S, Montplaisir J, Postuma RB. Rapid eye movement sleep behavior disorder and subtypes of Parkinson's disease. Mov Disord. 2012;27:996-1003.

9. Postuma RB, Gagnon JF, Vendette M, Charland K, Montplaisir J. REM sleep behaviour disorder in Parkinson's disease is associated with specific motor features. J Neurol Neurosurg Psychiatry. 2008;79:1117-21.

10. Wang G, Wan Y, Wang Y, Xiao Q, Liu J, Ma JF, Wang XJ, Zhou HY, Tan YY, Cheng Q, Chen SD. Visual hallucinations and associated factors in Chinese patients with Parkinson's disease: roles of RBD and visual pathway deficit. Parkinsonism Relat Disord. 2010;16:695-6.

11. Wang ZY, Zhang MY, Qiu GY, et al. Clinical application of Chinese edition of mini-mental state examination. Shanghai Arch Psychiatry. 1989;7(3):108-13.

12. Chahine LM, Xie SX, Simuni T, Tran B, Postuma R, Amara A, Oertel WH, Iranzo A, Scordia C, Fullard M, et al. Longitudinal changes in cognition in early Parkinson's disease patients with REM sleep behavior disorder. Parkinsonism Relat Disord. 2016;27:102-6. 
13. Benninger DH, Michel J, Waldvogel D, Candia V, Poryazova R, van Hedel HJ, Bassetti CL. REM sleep behavior disorder is not linked to postural instability and gait dysfunction in Parkinson. Mov Disord. 2010;25:1597-604.

14. Heister DS, Hayar A, Garcia-Rill E. Cholinergic modulation of GABAergic and glutamatergic transmission in the dorsal subcoeruleus: mechanisms for REM sleep control. Sleep. 2009;32:1135-47.

15. Hepp DH, Ruiter AM, Galis Y, Voorn P, Rozemuller AJ, Berendse HW, Foncke EM, van de Berg WD. Pedunculopontine cholinergic cell loss in hallucinating Parkinson disease patients but not in dementia with Lewy bodies patients. J Neuropathol Exp Neurol. 2013;72:1162-70.

16. Rinne JO, Ma SY, Lee MS, Collan Y, Roytta M. Loss of cholinergic neurons in the pedunculopontine nucleus in Parkinson's disease is related to disability of the patients. Parkinsonism Relat Disord. 2008;14:553-7.

17. Fling BW, Cohen RG, Mancini M, Nutt JG, Fair DA, Horak FB. Asymmetric pedunculopontine network connectivity in parkinsonian patients with freezing of gait. Brain. 2013;136:2405-18.

18. Geddes MR, Tie Y, Gabrieli JD, McGinnis SM, Golby AJ, Whitfield-Gabrieli S. Altered functional connectivity in Lesional Peduncular Hallucinosis with REM sleep behavior disorder. Cortex. 2016;74:96-106.

19. Boucetta S, Salimi A, Dadar M, Jones BE, Collins DL, Dang-Vu TT. Structural brain alterations associated with rapid eye movement sleep behavior disorder in Parkinson's disease. Sci Rep. 2016;6:26782

20. Tekriwal A, Kern DS, Tsai J, Ince NF, Wu J, Thompson JA. Abosch a.REM sleep behaviour disorder: prodromal and mechanistic insights for Parkinson's disease. J Neurol Neurosurg Psychiatry. 2017;88(5):445-51.

21. Lim JS, Shin SA, Lee JY, Nam H, Lee JY, Kim YK. Neural substrates of rapid eye movement sleep behavior disorder in Parkinson's disease. Parkinsonism Relat Disord. 2016;23:31-6.

22. Rolinski M, Szewczyk-Krolikowski K, Tomlinson PR, Nithi K, Talbot K, BenShlomo Y, Hu MT. REM sleep behaviour disorder is associated with worse quality of life and other non-motor features in early Parkinson's disease. J Neurol Neurosurg Psychiatry. 2014;85:560-6.

23. Vibha D, Shukla G, Goyal V, Singh S, Srivastava AK, Behari M. RBD in Parkinson's disease: a clinical case control study from North India. Clin Neurol Neurosurg. 2011;113:472-6.

24. Lee JE, Kim KS, Shin HW, Sohn YH. Factors related to clinically probable REM sleep behavior disorder in Parkinson disease. Parkinsonism Relat Disord. 2010;16:105-8.

25. Ozekmekci S, Apaydin H, Kilic E. Clinical features of 35 patients with Parkinson's disease displaying REM behavior disorder. Clin Neurol Neurosurg. 2005;107:306-9.

\section{Submit your next manuscript to BioMed Central and we will help you at every step:}

- We accept pre-submission inquiries

- Our selector tool helps you to find the most relevant journal

- We provide round the clock customer support

- Convenient online submission

- Thorough peer review

- Inclusion in PubMed and all major indexing services

- Maximum visibility for your research

Submit your manuscript at www.biomedcentral.com/submit

) Biomed Central 